\title{
Infected Anastomotic Pseudoaneurysm after Aortic Valve Replacement with Annular Enlargement
}

\author{
Runqian Sui, MD, Jie Zi, MD, Liangong Sun, MD, Decai Li, MD, Anbiao Wang, MD \\ Shandong Provincial Hospital, Shandong University, Jinan, China
}

\section{ABSTRACT}

Anastomotic pseudoaneurysm remains one of the main life-threatening complications of cardiac and thoracic aorta surgery. We report a rare case of infected pseudoaneurysm at the anastomotic line found during follow-up. Blood culture results suggested Enterococcus faecium infection. Transthoracic echocardiography and computed tomography scans revealed the presence of a pseudoaneurysm of the ascending aorta. The pseudoaneurysm was resected and the ascending aorta was reconstructed with an artificial vascular patch without complications. Reducing the anastomotic tension, with complete hemostasis at the anastomotic incision, is the most important means of preventing the formation of pseudoaneurysm.

\section{CASE REPORT}

A 30-year-old female was confirmed to have an infected pseudoaneurysm at the anastomosis of aortic incision after aortic valve replacement with annular enlargement performed in our hospital. She was first diagnosed with severe aortic valve stenosis with narrow aortic annulus. It was difficult to implant an appropriate-sized prosthetic valve in a routine fashion during the first surgery. We performed an annular enlargement in a modified Nick's procedure to prevent patient-prosthesis mismatch. A 19-mm St. Jude Medical Regent aortic valve was inserted into the enlarged annulus. The patient was discharged nine days after surgery.

The patient was treated with vancomycin and piperacillin sulbactam in a local hospital, and she suffered from persistent fever for 15 days, which began two months after the initial operation during the weight-process, but the anti-infective therapy effect was poor. Blood culture results suggested Enterococcus faecium infection. Considering the patient's history, the presence of a pseudoaneurysm of the ascending aorta was confirmed by transthoracic echocardiography (TTE) and computed tomography (CT) scans (Figure 1). TTE was used to identify the site of entry of the pseudoaneurysm. The pseudoaneurysm originated from the proximal

Received fanuary 9, 2019; accepted May1, 2019.

Correspondence: Anbiao Wang, MD, Department of Cardiovascular Surgery, Shandong Provincial Hospital affiliated to Shandong University, No. 324 fingwu Road, Finan 250021, China; (e-mail: $313823871 @ q q . c o m)$. ascending aortic root incision suture line. A chest CT scan showed a $5-\mathrm{cm}$ pseudoaneurysm originating from the anterior wall of the ascending aorta. The pseudoaneurysm was located at the anastomotic suture line, $5.4 \times 4.2 \mathrm{~cm}$ in diameter. Postoperative intrapericardial adhesions prevented the fatal rupture and led to a horseshoe-shape pseudoaneurysm extending around the ascending aorta root.

Considering that pseudoaneurysms can cause fatal rupture bleeding and death at any time, an emergency repair operation was performed via re-median sternotomy using the same skin incision as in the prior surgery without aneurysmal injury. After administering general anesthesia, the patient was placed in the supine position on the operating table. The right common femoral artery and vein were exposed, and then the patient was given anticoagulation treatment with heparin and arterial and vein catheterization. Cardiopulmonary bypass was then established after re-median sternotomy. Once the sternum was divided, cardiotomy suction was continued and careful adhesiolysis was performed. This allowed the borders of the pseudoaneurysm to be dissected from the posterior aspect of the sternum [Katsumata 2000]. The ascending aorta and superior vena cava were carefully exposed. Then the

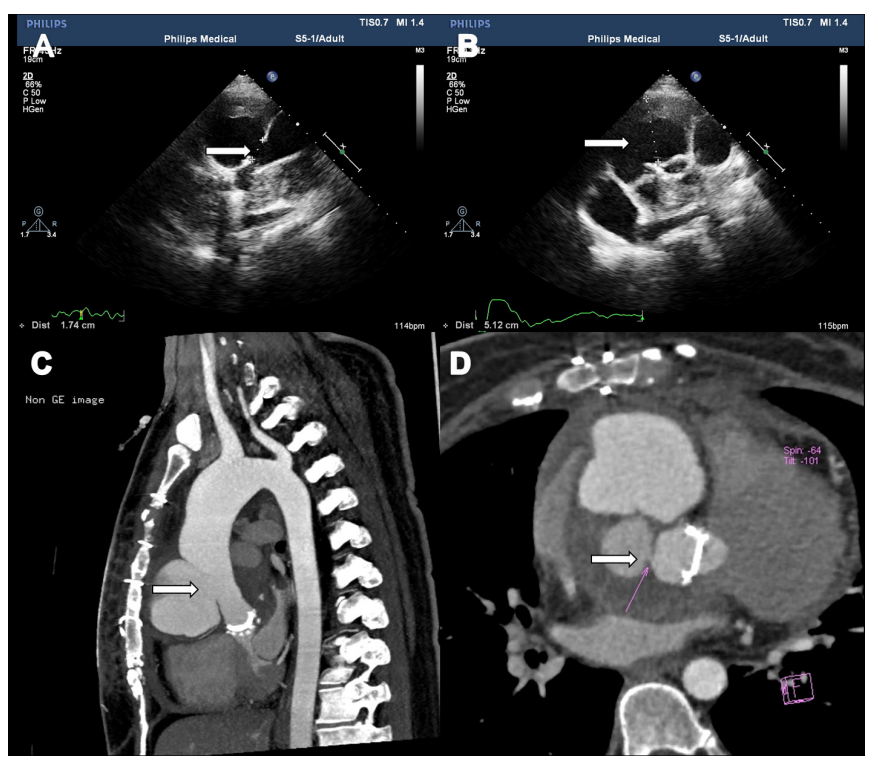

Figure 1. Site of entry into the anastomotic pseudoaneurysm, indicated by the white arrow (A, B). Horseshoe-shaped pseudoaneurysm extending around the ascending aorta trunk (C, D). 


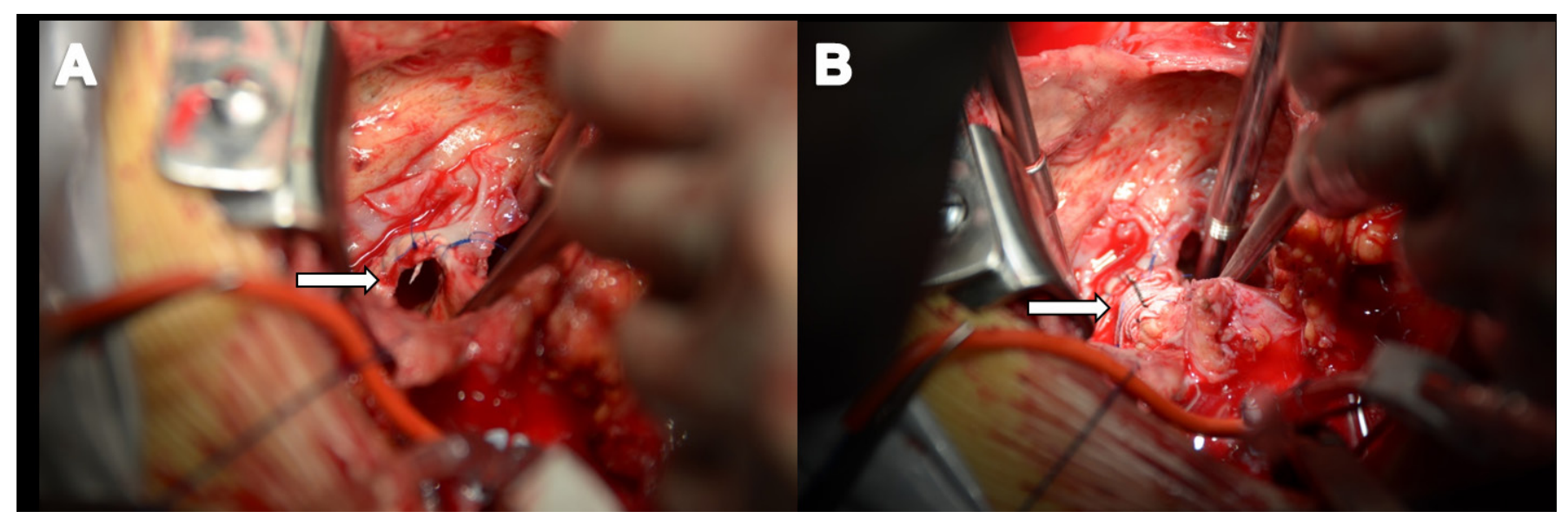

Figure 2. Intraoperative image showing the $2 \times 2 \mathrm{~cm}$ defect in the ascending aorta root (A). Intraoperative image showing the pseudoaneurysm had been repaired with artificial vascular patch $(B)$.

distal ascending aorta was dissected just before the innominate artery and a cross-clamp was applied. After the ascending aorta was clamped above the aneurysm, the pseudoaneurysm was opened, and cardiac arrest was achieved with antegrade infusion of cardioplegic solution. The pseudoaneurysm originated from the anastomotic line of the transverse incision of aortic root. It included pericardium tissue and thrombi, and an organized hematoma $5 \mathrm{~cm}$ in diameter was found overlapping the ascending aorta. A $2 \times 2 \mathrm{~cm}$ defect in the ascending aorta was repaired in two layers with 4-0 polyethylene sutures with PTFE artificial vascular patch (Figure 2). Cardiopulmonary bypass was then gradually tapered. The resected specimen was sent for culture and a diagnosis of Enterococcus faecium infection was made. The patient was discharged 28 days after surgery with anti-infective therapy; vancomycin was selected given the results of her drug sensitivity test.

\section{DISCUSSION}

Postoperative pseudoaneurysm of the ascending aorta is a somewhat rare postoperative complication, occurring in fewer than 3\% of all cardiac operations [Celiento 2014], and infected pseudoaneurysm is even rarer. Pseudoaneurysms can develop after cardiovascular thoracic surgery. Their risk factors include trauma, graft infections, dissected native aorta, and iatrogenic factors [Mauricio 2006]. The pathogenesis of the pseudoaneurysm creation is not yet totally clear. One possible mechanism is the gradual abatement of edema and swelling of the aortic wall after surgery, which causes the sutures to loosen. Another possible cause is laceration of the native aortic wall [Tanaka 2002], which occurs when the sutureholding capacity of the native aortic incision is insufficient and frictional force is brought to bear between the vascular wall and the anastomotic incision [Fehrenbacher 2007]. A pseudoaneurysm that forms rapidly has a thin and fragile wall and requires emergency surgery.

Once pseudoaneurysm is diagnosed, physicians must formulate an appropriate individual treatment before it enlarges
[Pietro 2013]. Conventional surgical treatment is considered the gold standard for most patients, but strict medical treatment can allow a delay or even prevent the need for surgery entirely. Methods for operating on anastomotic pseudoaneurysms are different from those for other aneurysms because of their different locations. Physicians must also choose the appropriate method of cardiopulmonary bypass. Peripheral cannulation, limited cardiopulmonary bypass, and circulatory arrest under deep hypothermia have been reported [Fehrenbacher 2007]. In this case, we exposed the peripheral vessels and intubated the patient using femoral arteriovenous catheterization, but we initiated cardiopulmonary bypass only after sternal reentry to reduce the period during which the patient would be under cardiopulmonary bypass. Traditional, conventional surgical repair of ascending aortic pseudoaneurysms is a widely accepted technique; however, certain patients are at considerable risk, including the elderly, those with coagulation issues, and those with a history of sternal reconstruction. The risk of surgery can be very high in patients with previous adhesion from repeat sternotomy. Endovascular and hybrid techniques involving artificial blood vessel stents have recently become widely used, with reports of thoracic endovascular aneurysm repair [Preventza 2014; Piffaretti 2016]. However, long-term follow-up results are still limited. The cover of the ostium forms a long landing area in the healthy segment of the aorta to prevent endoleakage and migration of the stent graft. For this reason, endovascular and hybrid therapy are not suitable alternative treatments for young or healthy patients who can undergo conventional surgery, given the risk of stent-graft late migration and considering the effect of age on progressive dilatation of thoracic aorta. Moreover, conventional surgery is extremely difficult in patients who have undergone endovascular treatment [Hiraoka 2015]. Conventional surgery provides the most durable repair and carries acceptable surgical risks for young patients in some cardiac surgery centers [Nizet 2016]. In this case, in which the patient was relatively young, the ascending aorta perforation was at the anastomotic line of the transverse aortotomy incision following the aortic valve replacement with annular enlargement, and the patient was also diagnosed 
with infective pseudoaneurysm, so we selected conventional surgery. The pseudoaneurysm was surrounded and controlled by closely-related thin mediastinal structures. The pseudoaneurysm was resected and the ascending aorta was reconstructed with an artificial vascular patch. The patient tolerated the procedure well and had no further adverse symptoms.

\section{Conclusion}

We experienced an extremely rare case of infected anastomotic pseudoaneurysm after aortic valve replacement with aortic annular enlargement. After observing this case, we propose the following measures: reducing the anastomotic tension, with complete hemostasis at the anastomotic incision, is the most important measure to prevent the formation of pseudoaneurysm; exposing peripheral vessels but not initiating cardiopulmonary bypass until after reentry might be an effective method for performing resternotomy for the treatment of ascending aortic pseudoaneurysms; patients should avoid excessive weight-loss exercises during the postoperative recovery period; in young patients or those with confirmed infections, it is advisable to select the conventional surgery first for the unforeseeable risks of endovascular and hybrid therapy.

\section{REFERENCES}

Celiento M, Saccocci M, De Martino A, et al. 2014. Stability of aortic annulus enlargement during aortic valve replacement using a bovine pericardial patch: an 18-year clinical, echocardiographic, and angio-computed tomographic follow-up. J Thorac Cardiovasc Surg 147:977-83.

Fehrenbacher JW, Hart DW, Huddleston E, et al. 2007. Optimal endorgan protection for thoracic and thoracoabdominal aortic aneurysm repair using deep hypothermic circulatory arrest. Ann Thorac Surg $83: 1041-6$

Hiraoka A, Chikazawa G, Tamura K, et al. 2015. Clinical outcomes of different approaches to aortic arch disease. J Vasc Surg 61:88e95.

Katsumata T, Moorjani N, Vaccari G, Westaby S. 2000. Mediastinal false aneurysm after thoracic aortic surgery. Ann Thorac Surg 70:547-52.

Villavicencio MA, Orszulak TA, Sundt III TM, et al. 2006. Thoracic aorta false aneurysm: what surgical strategy should be recommended? Ann Thorac Surg 82:81-9; discussion 89.

Nizet C, Van Damme H, Boesmans E, et al. 2016. Chronic false aneurysm after a healed rupture of the aortic isthmus: TEVAR, hybrid surgery, or open arch repair? Ann Vasc Surg 31:205.e11-6.

Malvindi PG, Cappai A, Raffa GM, et al. 2013. Analysis of postsurgical aortic false aneurysm in 27 patients. Tex Heart Inst J 40:274-80.

Piffaretti G, Galli M, Lomazzi C, et al. 2016. Endograft repair for pseudoaneurysms and penetrating ulcers of the ascending aorta. J Thorac Cardiovasc Surg 151:1606-14.

Preventza O, Henry MJ, Cheong BY, Coselli JS. 2014. Endovascular repair of the ascending aorta: when and how to implement the current technology. Ann Thorac Surg 97:1555-60.

Tanaka K, Makuuchi H, Naruse Y, et al. 2002. False aneurysm due to suture loosening after aortic arch replacement. Asian Cardiovasc Thorac Ann 10:346-8. 